\title{
COI and ND1 as DNA barcodes to identify Taenia hydatigena (Cestoda: Taeniidae) from canine small intestine on Hainan Island in China
}

\author{
Yajun Lu, Siqi Yang, Jingwen Liu, Xixi Lu, Qiuyu Zhao
}

Not peer-reviewed, not copy-edited manuscript.

Disclaimer on biological nomenclature and use of preprints

The preprints are preliminary versions of works accessible electronically in advance of publication of the final version. They are not issued for purposes of botanical, mycological or zoological nomenclature and are not effectively/validly published in the meaning of the Codes. Therefore, nomenclatural novelties (new names) or other nomenclatural acts (designations of type, choices of priority between names, choices between orthographic variants, or choices of gender of names) should NOT be posted in preprints. The following provisions in the Codes of Nomenclature define their status:

International Code of Nomenclature for algae, fungi, and plants (ICNafp)

Article 30.2: "An electronic publication is not effectively published if there is evidence within or associated with the publication that its content is merely preliminary and was, or is to be, replaced by content that the publisher considers final, in which case only the version with that final content is effectively published." In order to be validly published, a nomenclatural novelty must be effectively published (Art. 32.1(a)); in order to take effect, other nomenclatural acts must be effectively published (Art. 7.10, 11.5, 53.5, 61.3, and 62.3).

International Code of Zoological Nomenclature (ICZN)

Article: 21.8.3: "Some works are accessible online in preliminary versions before the publication date of the final version. Such advance electronic access does not advance the date of publication of a work, as preliminary versions are not published (Article 9.9)". 
2 COI and ND1 as DNA barcodes to identify Taenia hydatigena (Cestoda: Taeniidae)

3 from canine small intestine on Hainan Island in China

\section{Author names}

$5 \quad$ Siqi Yang ${ }^{1}$, Jingwen $\mathrm{Liu}^{1}$, Xixi Lu${ }^{1}$, Qiuyu Zhao ${ }^{1}$, Yajun $\mathrm{Lu}^{1 *}$

\section{Author affiliations}

$7 \quad 1$ Key Laboratory of Tropical Translational Medicine of Ministry of Education and

8 School of Tropical Medicine and Laboratory Medicine, Hainan Medical University,

9 Haikou, Hainan 571199, China.

\section{Conflict of interest statement}

11 The co-authors declare that they have no conflict of interest statement.

\section{*Correspondence author}

\section{Funding}

This study was supported by grants from the Natural Science Foundation of Hainan

17 Province (2019RC223).

\section{Ethics approval}

Not applicable. 


\section{Consent to participate}

All participants provided written informed consent.

\section{Consent for publication}

All participants obtained written informed consent for publication.

\section{Availability of data and material}

The data used to support the findings of this study are included in the article. All data are fully available without restriction.

\section{Code availability}

Not applicable.

\section{Authors' contributions}

Siqi Yang performed the experiments and wrote the manuscript. Jingwen Liu, Xixi Lu, and Qiuyu Zhao performed the experiments. Yajun Lu designed the study, performed revisions to the draft report, and approved the final manuscript.

\section{Acknowledgements}

The authors gratefully thank the Science and Technology Department of Hainan

Province for the financial support and Academician Workstation for material support. 


\section{Abstract}

DNA barcoding based on universal gene markers is a fast, accurate, and innovative approach for the molecular discrimination of species. Some species are particularly difficult to discriminate using a traditional morphological identification method because of severely damaged morphological features. In this study, cytochrome c oxidase subunit I (COI) and NADH dehydrogenase subunit 1 (ND1) were used as barcoding markers to distinguish Taenia hydatigena in dogs on the tropical island of Hainan. Therefore, geographic differentiation based on the COI and ND1 sequences amongst the specimens and other geographic isolates in GenBank was determined by calculating the genetic distances according to the Kimura 2-parameter (K2P) model and constructing a phylogenetic tree using the neighbour-joining (NJ) method. Barcoding gap, base composition, and base saturation were tested to assess the effectiveness of the barcoding marker COI and ND1 genes for specimen identification. In addition, we analysed the barcoding gap and saturation and performed molecular evolutionary analysis of the intraspecies and interspecies diversity of Taenia.

\section{Keywords}

Cytochrome c oxidase subunit I; DNA barcodes; NADH dehydrogenase subunit 1; Taenia hydatigena 


\section{Introduction}

With the development of molecular biology, it is increasingly common to identify species by combining molecular biology with genetic evolution analysis (Young et al. 2019). Molecular identification based on specific DNA sequence analyses has become a common technological tool used to identify new species and can make more accurate assessments within and between species biodiversity than other methods as well as serve as an effective supplement to traditional morphological identification (Valentini et al. 2009). DNA barcodes have been widely used in biological molecular identification and have great significance for uncovering biological diversity. DNA barcodes can be used to establish a large-scale classification system for biodiversity analysis through several species-specific DNA barcodes (Coissac et al. 2016). DNA barcodes also provide reliable species identification for all life stages and even for tissue debris (Hawlitschek et al. 2016).

The application of the barcode of the COI gene in the study of molecular biodiversity is increasing rapidly and continuously (Balech et al. 2018). There are approximately 4.5 million COI gene barcodes in GenBank and BOLD (Barcode of Life Data Systems), including different individuals across 250,000 species. It has become a common phenomenon to identify different animals by using the barcode of the COI gene (Thaler et al. 2016). In the identification of some species, the COI gene is more suitable for identifying individuals (Laopichienpong et al. 2016). The COI gene may prove to be valuable in the identification of the early developmental stage of species (Jaakko et al. 2016). 
T. hydatigena is a zoonotic tapeworm species. Cysticercus is a larva that can be widely parasitized in domestic animals with a high infection rate. Hainan is a high incidence area of parasitic diseases. Taenia is a highly diverse population, and using morphological methods alone may fail to identify some parasites accurately (Ulziijargal et al. 2019). In 2013, Minoru and colleagues constructed molecular phylogeny using mitochondrial genes to explain the genetic relationship among different Taenia (Minoru et al. 2013). Since two gene barcodes have been proven to have better resolution (Pennisi 2007), we use two genes, COI and ND1, as barcodes to reflect the diversity of $T$. hydatigena (Cestoda: Taeniidae) on Hainan Island, and we analyse the molecular evolution of Taenia using molecular genetics.

\section{Materials and methods}

\section{Sample collection and genomic DNA extraction}

An adult worm was obtained from the canine small intestine. The fresh worm was washed in physiological saline and stored in $70 \%$ ethanol until use. Approximately 2-3 millimetres of worm was cut with a scalpel and washed three times with $\mathrm{ddH}_{2} \mathrm{O}$. The small piece of worm tissue was digested in $100 \mu \mathrm{L}$ Solution AB and $25 \mu \mathrm{L}$ AD Buffer (Bioteke, Beijing, China) for $10 \mathrm{~min}$ at room temperature followed by $5 \mathrm{~min}$ at $95^{\circ} \mathrm{C}$ and was subsequently added to $100 \mu \mathrm{L}$ Solution AC (Bioteke) to extract the genomic DNA.

\section{PCR amplification and sequencing}


reaction (PCR). The primer sequences for $\mathrm{COI}$ were the forward primer 5'-TTTTTTGGGCATCCTGAGGTTTAT-3' and reverse primer

5'-TAAAGAAAGAACATAATGAAAATG-3'. The primer sequences for ND1 were the forward primer 5'-AGATTCGTAAGGGGCCTAATA-3' and reverse primer 5'-ACCACTAACTAATTCACTTTC-3'. Amplification reactions contained $10 \mu \mathrm{L}$ of $2 \times$ Power Taq PCR MasterMix (Bioteke), $4 \mu \mathrm{L}$ of dd $\mathrm{d}_{2} \mathrm{O}, 1 \mu \mathrm{L}$ of each forward and reverse primer $(10 \mu \mathrm{M})$, and $4 \mu \mathrm{L}$ of template DNA $(0.002 \mu \mathrm{g} / \mu \mathrm{L})$. The cycling conditions were as follows: $94{ }^{\circ} \mathrm{C}$ for $3 \mathrm{~min}, 35$ cycles of $94{ }^{\circ} \mathrm{C}$ for $1 \mathrm{~min}, 55^{\circ} \mathrm{C}$ for 1 min and $72{ }^{\circ} \mathrm{C}$ for $1 \mathrm{~min}$, followed by a final step of $72{ }^{\circ} \mathrm{C}$ for $5 \mathrm{~min}$. PCR products were purified using a FastPure Gel DNA Extraction Mini Kit (Vazyme Biotech, Nanjing, China) and sequenced by the Sanger sequencing method (BGI, Beijing, China).

\section{Molecular evolution analysis}

The sequences generated were assembled and refined manually to obtain final high-quality sequences using ApE software v2.0.61

(http://jorgensen.biology.utah.edu/wayned/ape). Multiple sequence alignment was conducted for the sequences of the samples for COI and ND1, the selected sequences of 29 geographic isolates for COI, and 21 geographic isolates for ND1 from the GenBank database using the Basic Local Alignment Search Tool (BLAST) of NCBI (National Center for Biotechnology Information) (https://blast.ncbi.nlm.nih.gov/Blast.cgi). The phylogenetic trees were constructed using the neighbour-joining (NJ) algorithm. The intraspecific and interspecific genetic 
119

120

distances were calculated using the Kimura 2-parameter (K2P) model, and the nucleotide composition, nucleotide frequencies, nucleotide pair frequencies, and transition/transversion ratios were analysed using MEGA-X software.

\section{Haplotype diversity analysis}

Haplotype diversity (Hd) was evaluated using DNA Sequence Polymorphism (DNAsp)

v6.0 software by calculating the number of segregating sites (S), the average number of nucleotide differences per site (pi), and the total number of mutations.

\section{Effectiveness assessments of the barcoding markers}

The barcoding gaps of the markers for COI and ND1 genes were detected using automatic barcode gap discovery (ABGD). The values of substitution saturation were calculated using Data Analysis in Molecular Biology and Evolution (DAMBE) v7.2.14 software.

\section{Results}

\section{Morphological characters}

The adult worm was opalescent-yellowish and measured $122.4 \mathrm{~cm}$ in length. The strobila had 250-300 proglottids. The immature proglottids were wider than longer, and the mature proglottids were longer than wide at 10-14 $\mathrm{mm}$ long and 4-5 mm wide. A mature proglottid had an ovary and 600-700 testes. The uterus, filled with eggs, had 5-10 uterine branches with smaller branches in a gravid proglottid.

Each genital pore was unilateral and irregularly alternating. The eggs were oval in 
139

140

141

142

143

144

145

146

147

148

149

150

151

152

153

154

155

156

157

158

shape and approximately 36-39 $\mathrm{mm}$ in size, containing an oncosphere (Figure 1).

\section{Phylogenetic characteristics}

A 422-bp fragment of the COI gene was successfully amplified, and a 385-bp fragment was retained after manual editing. Additionally, a 502-bp fragment of the COI gene was successfully amplified, and a 471-bp fragment was retained after manual editing. The phylogenetic tree of the COI sequence (Figure 2) showed that $T$. hydatigena Hainan isolates had the closest relationship with T. hydatigena from Iran (Table 1), and the phylogenetic tree of the ND1 sequence (Figure 3) showed that $T$. hydatigena Hainan isolates had the closest relationship with T. hydatigena from Kenya (Table 2). The nucleotide sequence homology among the Taenia hydatigera was very similar, showing high homogeneity. For the ND1 gene, the genetic relationship between Taenia hydatigera and Taenia saginata was the furthest.

\section{Genetic diversity analyses}

A total of 15 haplotypes were obtained from the sequence alignment of 29 isolates based on the COI gene: the haplotype diversity was 0.8276 , the nucleotide diversity pi was 0.07769 , and the number of segregating sites was 130 . The average content of the
$\mathrm{A}, \mathrm{T}, \mathrm{C}$, and $\mathrm{G}$ bases from the $\mathrm{COI}$ gene sequences was $22.3 \%, 44.7 \%, 9.6 \%$, and $23.5 \%$, respectively, and the GC content was $33.1 \%$. The COI gene contained 81 variable sites. The transitional pairs (si) were 10, and the transversional pairs (sv) were 13 with a si/sv ratio of 0.77 . 
160

161

162

163

164

165

166

167

168

169

170

171

172

173

174

175

176

177

178

179

180

based on the ND1 gene: the haplotype diversity was 0.9381 , the nucleotide diversity pi was 0.12544 , and the number of segregating sites was 260 . The average content of

$\mathrm{A}, \mathrm{T}, \mathrm{C}$, and $\mathrm{G}$ bases from ND1 gene sequences was $19.7 \%, 51.6 \%, 6.5 \%$, and $22.3 \%$, respectively, and the GC content was $28.8 \%$. The ND1 gene contained 94 variable

sites. The transitional pairs (si) were 10, and the transversional pairs (sv) were 11 with a si/sv ratio of 0.91 . The transversional pairs of the COI gene turned into a straight upward trend with increasing genetic distance, and when the base conversion proportion was 3\%, the platform period appeared. For ND1, both transitional pairs and transversional pairs had a platform period. The mutation of the ND1 gene reached a saturation state (Figure 4).

\section{Effectiveness of the barcoding markers}

The COI gene had obvious DNA barcoding gaps. The DNA barcoding gaps of the COI gene were 0.03-0.12 and 0.20-0.30, and the DNA barcoding gap of the ND1 gene was 0.45-1.25 (Figure 5). The COI and ND1 genes both showed DNA barcoding gaps within and between populations, and they had a certain genetic interval; therefore, they could be used to distinguish species.

We used MEGA-X to calculate the genetic distance within and between different tapeworms in COI and ND1. For COI, the maximum genetic distance was between Taenia saginata and Taenia taeniaformis. The genetic distance between Taenia saginata and other worms was far. For ND1, the results showed that the genetic distance between Taenia hydatigera and Taenia saginata was the largest, and they had 
181

182

the furthest genetic relationship. The histogram of distance shows that the COI sequence set is divided into 8 distinct groups, and the ND1 sequence set is divided into 7 distinct groups.

\section{Discussion}

T. hydatigera is an endoparasite that causes human and animal infections. Adult worms inhabit the small intestine of canines, wolves, and other canids. The larvae disseminate to the livers, lungs, and various other organs of livestock and wildlife. Humans usually develop infections from ingesting food and water contaminated by parasite eggs in canine faeces. In 2003, Hebert et al. first proposed the term DNA barcoding (Hebert et al. 2003) as a technique to identify organisms. DNA barcoding is an efficient tool for distinct species (DeSalle et al. 2005; Frézal and Leblois 2008). Accumulating evidence shows that the COI gene can serve as the core of a global bioidentification system for animals. The COI gene can distinguish species at the DNA level. A DNA barcoding technique based on the molecular evolution of the COI gene is a reliable classification method. The COI gene is a general DNA barcoding marker; nevertheless, the molecular identification rate of sibling species or related species of ecogeographical groups is relatively low. In addition, DNA barcoding markers of multiple genes have better discriminability than a single gene as a DNA barcoding marker. An outstanding DNA barcoding marker requires that there is an obvious barcoding gap between intra- and interspecific distances. The average interspecific distances were more than 10-fold larger than the average intraspecific distances (Paul et al. 2004). Therefore, we used the COI and ND1 genes as DNA 
203

204

barcoding markers to identify the species of the worm and analysed the phylogenetic relationships of the specimen and different geographic isolates in this study. The $T$. hydatigena Hainan isolate had the closest phylogenetic relationship with the $T$. hydatigena Chabahar isolate from Iran based on the COI gene. The T. hydatigena Hainan isolate had the closest phylogenetic relationship with the T. saginata Kenya isolate from Iran based on the ND1 gene. The phylogenetic tree and genetic distance showed that the genetic distance between Taenia saginata and other worms was far. Two different genes had the same result. Through the existence of a barcode gap, we could automatically classify species sequences. The existence of a barcode gap proved that species can be distinguished effectively (Puillandre et al. 2011). The COI gene and the ND1 gene both showed an obvious barcode gap. There were two barcode gaps in the COI gene. These results suggest that the identification effect of the COI gene is better in species and interspecies of Taenia.

After the analysis of bases, we found that the haplotype diversity, base polymorphism, and polymorphic isolate sites of the ND1 gene were all high. In addition, the mutation of the ND1 gene reached a saturation state. Mitochondrial genes often have mutation saturation and a rapid mutation rate, so in the process of species formation, a base site may have multiple mutations, which will lead to a reduction in genetic distance when comparing distant species (Blouin et al. 1998). Using the COI gene may be more accurate and suitable.

In conclusion, it is extremely important to use DNA barcodes for species identification, especially for species that are morphologically similar and difficult to 
distinguish by morphological methods. At present, due to the wide application of DNA barcodes, many researchers have conducted research on DNA barcodes and have proposed methods with high precision and calculation efficiency to improve the classification and recognition of barcodes (Goldstein and DeSalle 2011). DNA barcode identification of species has a good perspective. The principle of DNA barcodes is to select highly conserved DNA coding regions or noncoding region fragments with subtle variation at the evolutionary level to identify species, but it is difficult to find such general standard sequence fragments. In the process of species identification, we should also consider the mutation saturation of bases and properly use the genetic information analysis of base transversion to improve accuracy. Our study is relevant for its identification of T. hydatigena in Hainan. Moreover, with the development of DNA barcode research, DNA barcode technology will significantly advance the biodiversity research field.

\section{References}

Balech B, Sandionigi A, Manzari C, Trucchi E, Saverio V (2018) Tackling critical parameters in metazoan meta-barcoding experiments: A preliminary study based on coxI DNA barcode. PeerJ 6: e4845. https://doi.org/10.7717/peerj.4845

Blouin MS, Yowell CA, Courtney CH, Dame JB (1998) Substitution bias, rapid saturation, and the use of mtDNA for nematode systematics. Molecular Biology \& Evolution 12: 1719-1727. https://doi.org/10.1093/oxfordjournals.molbev.a025898 
247

248

Coissac E, Hollingsworth PM, Lavergne S, Taberlet P (2016) From barcodes to genomes: extending the concept of DNA barcoding. Molecular Ecology 25: 1423-1428. https://doi.org/10.1111/mec.13549

DeSalle R, Egan MG, Siddall M (2005) The unholy trinity: taxonomy, species delimitation and DNA barcoding. Philosophical Transactions of the Royal Society B Biological Sciences 360: 1905-1916. https://doi.org/10.1098/rstb.2005.1722

Frézal L, Leblois R (2008) Four years of DNA barcoding: current advances and prospects. Infection Genetics \& Evolution 8: 727-736. https://doi.org/10.1016/j.meegid.2008.05.005

Goldstein PZ, DeSalle R (2011) Integrating DNA barcode data and taxonomic practice: determination, discovery, and description. Bioessays 33: 135-147. https://doi.org/10.1002/bies.201000036

Hawlitschek O, Morinière J, Dunz A, Franzen M, Rödder D, Glaw F, Haszprunar G (2016) Comprehensive DNA barcoding of the herpetofauna of Germany. Molecular Ecology Resources 16: 242-253. https://doi.org/10.1111/1755-0998.12416

Hebert PDN, Cywinska A, Ball SL, deWaard JR (2003) Biological identifications through DNA barcodes. Proceedings Biological sciences 270: 313-321. https://doi.org/10.1098/rspb.2002.2218

Jaakko LOP, Kahanpää J, Mutanen M (2016) DNA Barcodes for the Northern European Tachinid Flies (Diptera: Tachinidae). Plos One 11: e0164933. 
Laopichienpong N, Muangmai N, Supikamolseni A, Twilprawat P, Chanhome L, Suntrarachun S, Peyachoknagul S, Srikulnath K (2016) Assessment of snake DNA barcodes based on mitochondrial COI and Cytb genes revealed multiple putative cryptic species in Thailand. Gene 594: 238-247. https://doi.org/10.1016/j.gene.2016.09.017

Minoru, Nakao, and, Antti, Lavikainen, and, Takashi, Iwaki, and, Voitto (2013) Molecular phylogeny of the genus Taenia (Cestoda: Taeniidae): Proposals for the resurrection of Hydatigera Lamarck, 1816 and the creation of a new genus Versteria. International Journal for Parasitology 43: 427-437. https://doi.org/10.1016/j.ijpara.2012.11.014

Paul DNH, Penton EH, Burns JM, Janzen DH, Hallwachs W (2004) Ten Species in One: DNA Barcoding Reveals Cryptic Species in the Neotropical Skipper Butterfly Astraptes fulgerator. Proceedings of the National Academy of Sciences of the United States of America 101: 14812-14817. https://doi.org/10.1073/pnas.0406166101

Pennisi E (2007) Taxonomy. Wanted: a barcode for plants. Science 318: 190-191. https://doi.org/10.1126/science.318.5848.190

Puillandre N, Lambert A, Brouillet S, Achaz G (2011) ABGD, Automatic Barcode Gap Discovery for primary species delimitation. Molecular Ecology 21: 1864-1877. https://doi.org/10.1111/j.1365-294X.2011.05239.x

Thaler, David S, Stoeckle, Mark Y (2016) Bridging two scholarly islands enriches 
both: COI DNA barcodes for species identification versus human mitochondrial variation for the study of migrations and pathologies. Ecology and Evolution 6: 6824-6835. https://doi.org/10.1002/ece3.2394

Ulziijargal G, Yeruult C, Khulan J, Gantsetseg C, Wandra T, Yamasaki H, Myadagsuren N (2019) Molecular identification of Taenia hydatigena and Mesocestoides species based on copro-DNA analysis of wild carnivores in Mongolia. International Journal for Parasitology: Parasites and Wildlife 11: 72-82. https://doi.org/10.1016/j.ijppaw.2019.12.004

Valentini A, Pompanon F, Taberlet P (2009) DNA barcoding for ecologists. Trends in Ecology \& Evolution 24: 110-117. https://doi.org/10.1016/j.tree.2008.09.011 Young MR, Proctor HC, deWaard JR, Hebert PDN (2019) DNA barcodes expose unexpected diversity in Canadian mites. Molecular Ecology 28: 5347-5359. https://doi.org/10.1111/mec.15292 

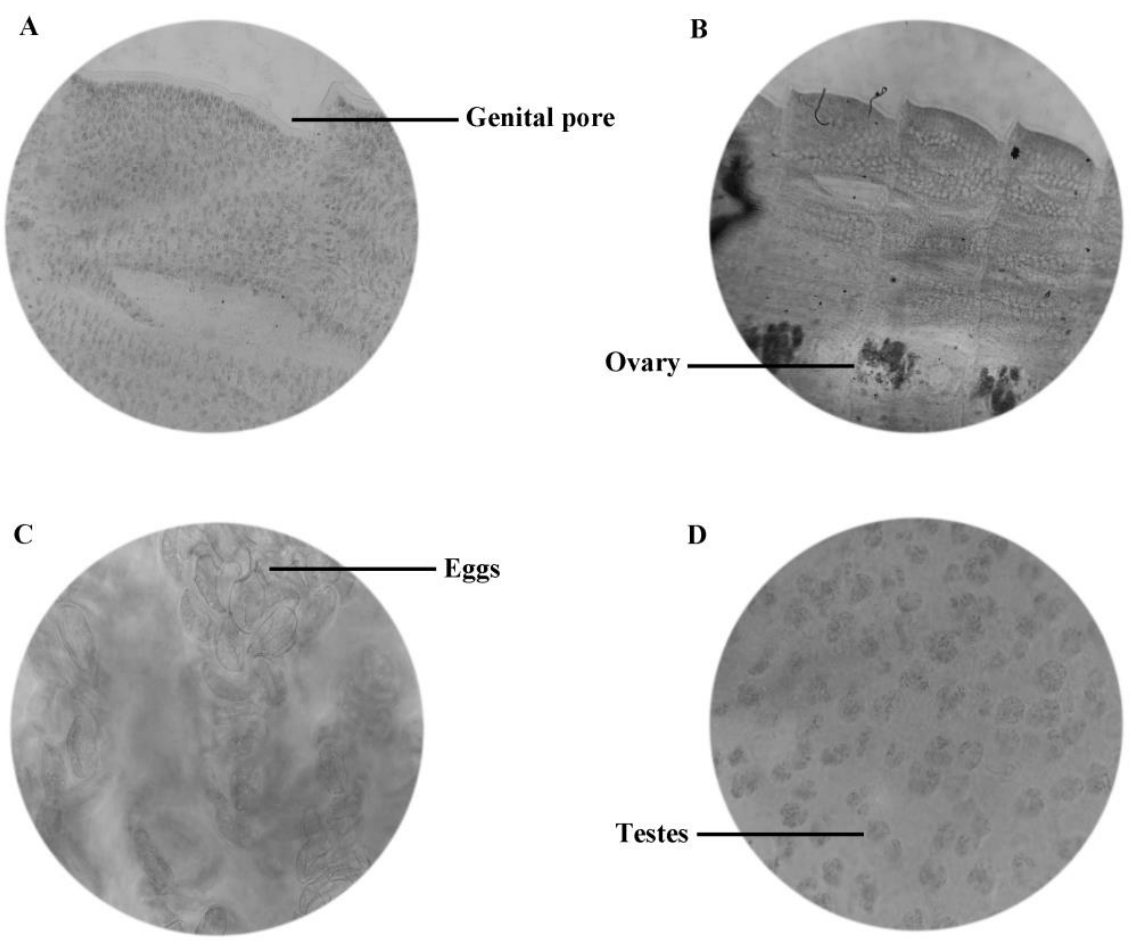

304

305 Figure 1. T. hydatigena tissues were made transparent in xylene.

306 The microscope magnification is $\times 100$ in $1-\mathrm{A}, \mathrm{C}$, and $\mathrm{D}$.

307 The microscope magnification is $\times 40$ in $1-\mathrm{B}$. 


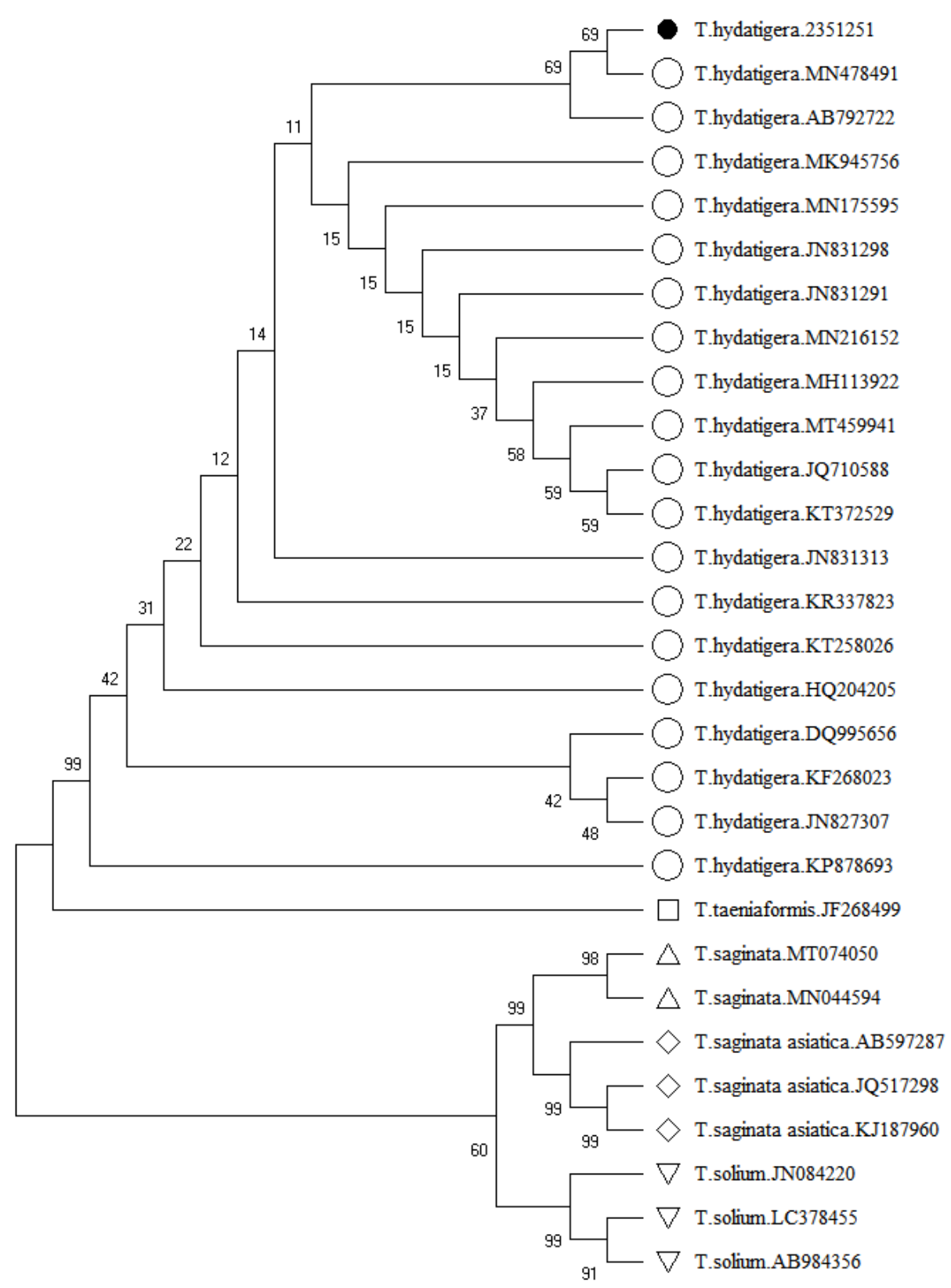

309 Figure 2. The COI NJ phylogenetic tree is shown for 29 different geographic isolates.

310 Different symbols indicate different species. There are five different kinds of Taenia

311 spp. The black circle denotes the T. hydatigena (Cestoda: Taeniidae) in Hainan

312 identified by the COI gene. 


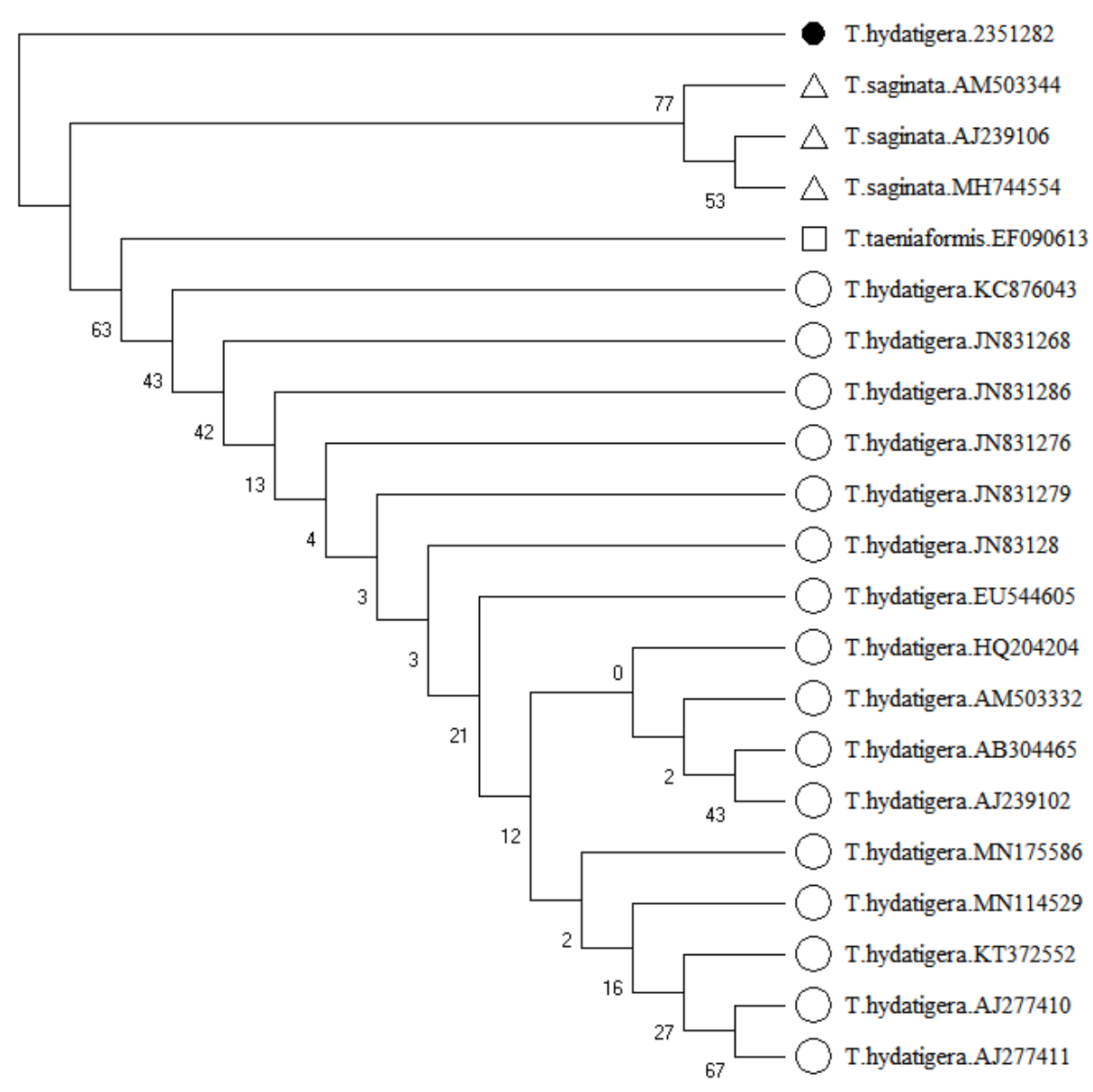

314 Figure 3. The ND1 NJ phylogenetic tree is shown for 21 different geographic isolates.

315 Different symbols indicate different species. There are three different kinds of Taenia spp. The black circle denotes the T. hydatigena in Hainan identified by the ND1 gene. 

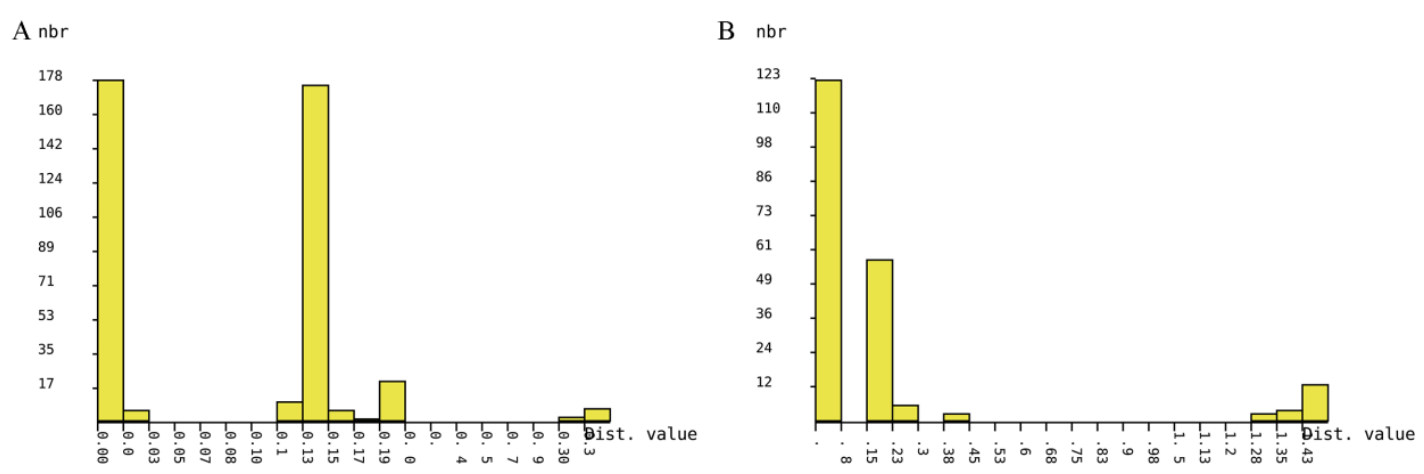

318 Figure 4. Results of barcoding gap analysis of 29 COI genes (A) and 21 ND1 genes

319 (B) generated by ABDG. The abscissa represents the distance value, and the ordinate represents the numbers. Both genes showed an obvious barcoding gap. 

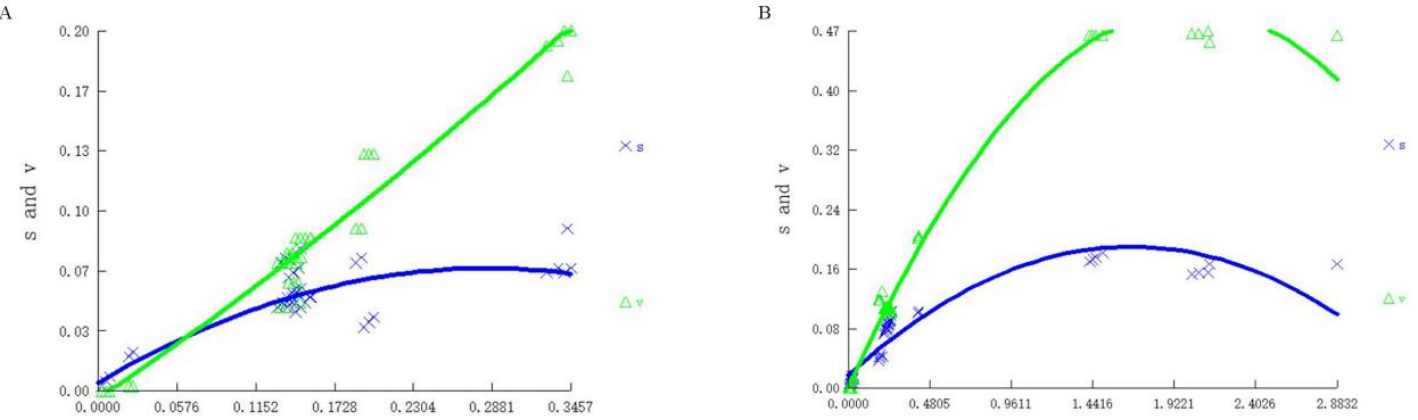

322 Figure 5. Results of base saturation analysis of 29 COI genes (A) and 21 ND1 genes

323 (B). The abscissa indicates genetic distance, and the ordinate indicates base

324 substitution frequency. 
325 Table 1. The COI gene of the 29 different geographic isolates

\begin{tabular}{|c|c|c|c|c|c|}
\hline 326 & GenBank ID & Species & Source & Query Cover (\%) & Per.Ident(\%) \\
\hline 327 & MN478491 & Taenia hydatigera & Chabahar & 98 & 99.52 \\
\hline 328 & AB792722 & Taenia hydatigera & Mongolia & 98 & 99.52 \\
\hline 329 & HQ204205 & Taenia hydatigera & China & 98 & 99.28 \\
\hline 330 & DQ995656 & Taenia hydatigera & India & 98 & 99.28 \\
\hline 331 & MN216152 & Taenia hydatigera & Zambia & 96 & 99.26 \\
\hline 332 & MK945756 & Taenia hydatigera & Ghana & 98 & 99.04 \\
\hline 333 & MN175595 & Taenia hydatigera & Nigeria & 98 & 99.04 \\
\hline 334 & JN831298 & Taenia hydatigera & China & 98 & 99.04 \\
\hline 335 & JN831291 & Taenia hydatigera & China & 98 & 99.04 \\
\hline 336 & MH113922 & Taenia hydatigera & Iraq & 93 & 99.75 \\
\hline 337 & KR337823 & Taenia hydatigera & Kashmir & 98 & 98.80 \\
\hline 338 & JN831313 & Taenia hydatigera & China & 98 & 98.80 \\
\hline 339 & MT459941 & Taenia hydatigera & Pakistan & 91 & 100 \\
\hline 340 & KT258027 & Taenia hydatigera & China & 95 & 99.26 \\
\hline 341 & JQ710588 & Taenia hydatigera & Iran & 91 & 100 \\
\hline 342 & KT372529 & Taenia hydatigera & Italy & 91 & 100 \\
\hline 343 & KP878693 & Taenia hydatigera & Peru & 94 & 99.25 \\
\hline 344 & KF268023 & Taenia hydatigera & Turkey & 97 & 99.03 \\
\hline 345 & JN827307 & Taenia hydatigera & Turkey & 95 & 99.26 \\
\hline 346 & JN084220 & Taenia solium & Africa & 98 & 87.08 \\
\hline 347 & LC378455 & Taenia solium & Indonesia & 96 & 86.80 \\
\hline 348 & AB984356 & Taenia solium & China & 96 & 86.80 \\
\hline 349 & MN337881 & Taenia saginata & Slovakia & 97 & 87.38 \\
\hline 350 & МT074050 & Taenia saginata & Cambodia & 96 & 87.50 \\
\hline 351 & MN044594 & Taenia saginata & China & 98 & 87.05 \\
\hline 352 & AB597287 & Taenia saginata asiatica & Japan & 96 & 86.76 \\
\hline 353 & JQ517298 & Taenia saginata asiatica & Thailand & 96 & 86.76 \\
\hline
\end{tabular}


356 
357 Table 2 The ND1 gene of the 21 different geographic isolates

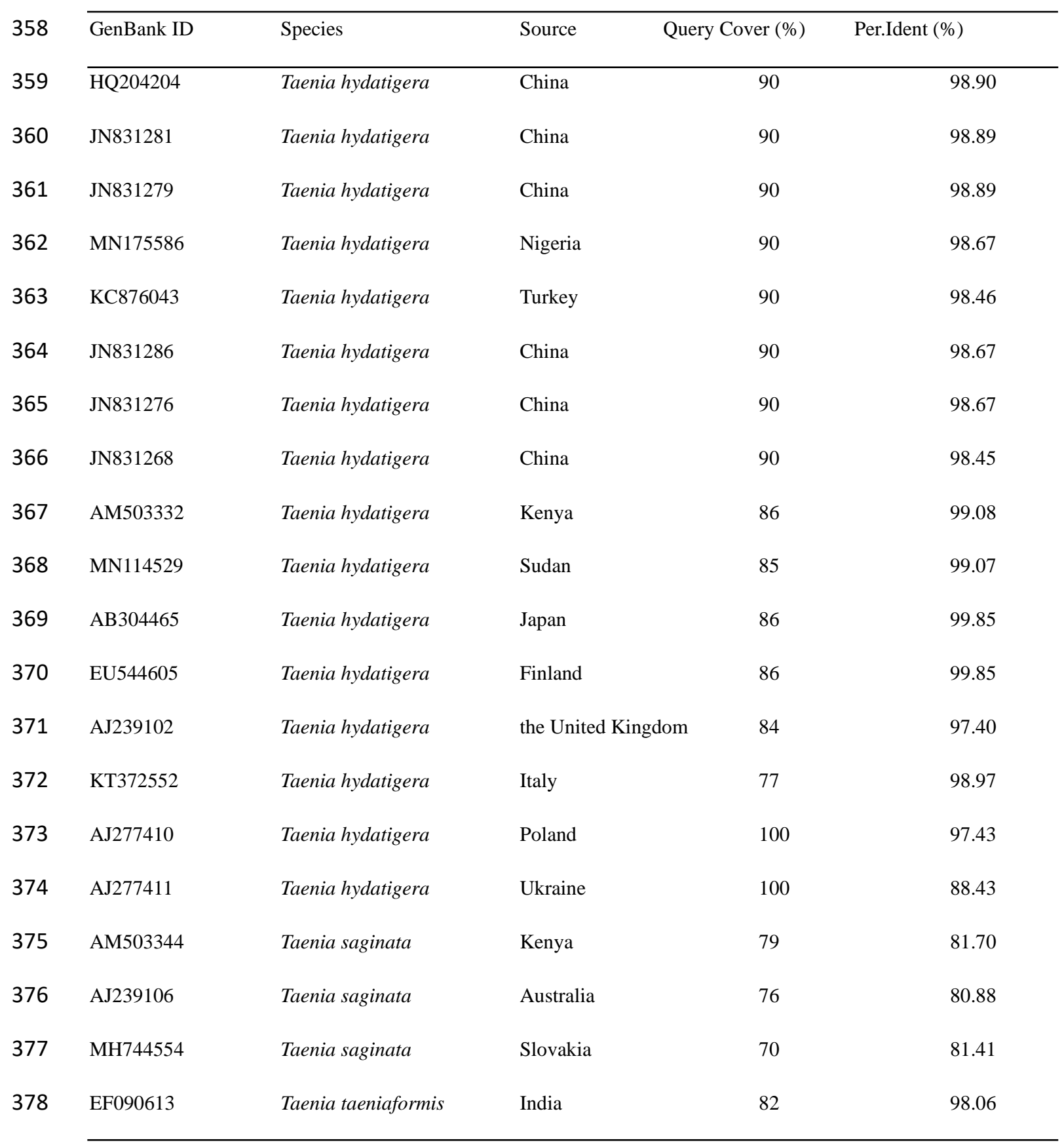

